\title{
Kinetic and Spectroscopic Characterisation of Highly Reactive Methanesulfonates. Leaving Group Effects for Solvolyses and Comments on Geminal Electronic Effects influencing $S_{\mathbb{N}} 1$ Reactivity
}

\author{
T. William Bentley,", Manfred ChristJ,"b Ralf Kemmer,b Gareth Llewellyn* and John E. Oakley" \\ - Department of Chemistry, University College of Swansea, Singleton Park, Swansea, Wales, \\ UK SA2 8PP \\ b Institut für Organische Chemie. Universität Würzburg. Am Hubland, D-97074, Würzburg. Germany
}

\begin{abstract}
Highly reactive methanesulfonates (mesylates, ROMs) have been prepared from 1-phenylethanol, cyclohex-2-en-1-ol, diphenylmethanol and p-methoxybenzyl alcohol by treatment with methanesulfonyl chloride and triethylamine in dichloro- or trichloro-methane at -20 to $0^{\circ} \mathrm{C}$. The mesylates, characterised in solution by ${ }^{1} \mathrm{H}$ and ${ }^{13} \mathrm{C}$ NMR at $-20^{\circ} \mathrm{C}$, were obtained in satisfactory purity (ca. $95 \%$ ) in cold solutions but they decomposed by reaction with chloride, triethylamine or the parent alcohol. Rate constants for solvolyses in aqueous acetone and aqueous ethanol have been determined by a fast response conductimetric method. Product selectivities for solvolyses of $p$ methoxybenzyl mesylate in aqueous ethanol and methanol at $0^{\circ} \mathrm{C}$ have been determined by $\mathrm{HPLC}$. From additional new or literature kinetic data for solvolyses of corresponding bromides, chlorides and $p$-nitrobenzoates (OPNB), $\mathrm{Br} / \mathrm{Cl}, \mathrm{OMs} / \mathrm{Br}$ and $\mathrm{OMs} / \mathrm{OPNB}$ rate ratios were calculated; the results are consistent with electronic effects stabilising the carbocationic transition states and increasing $\mathrm{OMs} / \mathrm{Br}$ rate ratios for these $S_{N} 1$ solvolyses; none of the evidence supports a geminal electronic effect on $\mathrm{Br} / \mathrm{Cl}$ rate ratios (e.g. caused by stabilisation of the initial state in $p$. methoxybenzyl chloride). Steric effects on ester/halide rate ratios for solvolyses of tertiary substrates are confirmed. Relative rates over a $10^{18}$ range for ester and halide leaving groups are evaluated for solvolyses of 1 -phenylethyl substrates in $80 \%$ ethanol-water, updating previous work by Noyce et al. (1972).
\end{abstract}

Rapid-injection kinetic methods have recently been applied to solvolyses of highly reactive sulfonates, ${ }^{1-4}$ including 1 -adamantyl trifluoromethanesulfonate synthesised from 1-adamantyl halides and silver trifluoromethanesulfonate in an alkane solvent. ${ }^{1}$ Similar methods were earlier used to synthesise diphenylmethyl, ${ }^{5,6}$ tert-butyl ${ }^{6}$ and 1-phenylethyl toluene-psulfonates (tosylates), ${ }^{6}$ all of which could be isolated at low temperatures. $p$-Methoxybenzyl tosylate has also been synthesised, ${ }^{6.7}$ but it is not well characterised because it is extremely reactive (it polymerises slowly even at $-60^{\circ} \mathrm{C}$ ). ${ }^{7}$

An alternative procedure for the synthesis of methanesulfonates (mesylates) is to treat an alcohol with methanesulfonyl chloride and triethylamine. ${ }^{8,9}$ An alcohol is usually a more accessible and reliable starting material than a halide, particularly as is often the case, rearrangements or loss of stereochemical configuration are possible: e.g. for trimethylsilyl2-norbornyl ${ }^{2}$ and tricyclo[3.1.0.0 $\left.{ }^{2.6}\right]$ hex-3-y] derivatives. An important advantage of mesylates over tosylates is their more rapid rates of dissolution in solvolytic media, which greatly facilitates kinetic studies in aqueous media.

We now report the first NMR characterisation of $p$ methoxybenzyl 1 and cyclohex-2-en-1-yl 2 mesylates, and their solvolysis rate constants. Kinetic data are also reported for solvolyses of diphenylmethyl 3, and 1-phenylethyl 4 mesylates. By comparing these data with rate constants for solvolyses of corresponding halides, predictions are made about the scope of the synthetic method for these highly reactive mesylates. Available literature on leaving group effects on solvolyses is extended with additional mesylate/p-nitrobenzoate and mesylate/bromide rate ratios, and their relevance to our understanding of geminal electronic effects on $S_{N} l$ reactivity will be discussed. Solvolyses of 1-phenylethyl substrates provide the most comprehensive scale of leaving group effects in $S_{N} 1$ reactions, ${ }^{10}$ and we are now better able to define the relative<smiles>COc1ccc(CCOc2ccccc2)cc1</smiles><smiles>CO[R]OC(c1ccccc1)c1ccccc1</smiles>

3<smiles>BrC1C=CCCC1</smiles><smiles>COc1ccc(C([O+])(C(F)(F)F)C(F)(F)F)cc1</smiles><smiles>CO[Ge](C)(O)OC1C=CCCC1</smiles>

2<smiles>CO[R]O[C@H](C)c1ccccc1</smiles>

4

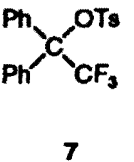

reactivities of sulfonates in comparison with less reactive leaving groups.

\section{Results}

Rate constants for solvolyses of the following mesylates are tabulated: p-methoxybenzyl 1, Table 1; cyclohex-2-en-1-yl 2, Table 2; diphenylmethyl 3, Table 3; 1-phenylethyl 4, Table 4. 
Table 1 Rate constants $(k)$ for solvolyses of $p$-methoxybenzyl mesylate $1^{\bullet}$

\begin{tabular}{|c|c|c|c|c|}
\hline Solvent ${ }^{b}$ & $T /{ }^{\circ} \mathrm{C}$ & $k / \mathrm{s}^{-1}$ & $\begin{array}{l}\Delta H^{\ddagger} / \\
\mathrm{kcal} \mathrm{mol}^{-1 \mathrm{c}}\end{array}$ & $\begin{array}{l}\Delta S^{t} / \mathrm{cal}^{-1} \\
\mathrm{~mol}^{-1} \mathrm{~K}^{-1 \mathrm{c}}\end{array}$ \\
\hline $\begin{array}{l}\text { EtOH } \\
90 \% \text { EtOH } \\
80 \% \text { EtOH } \\
90 \% \text { Acetone } \\
80 \% \text { Acetone } \\
70 \% \text { Acetone } \\
60 \% \text { Acetone }\end{array}$ & $\begin{array}{r}0.0 \\
25.0 \\
-19.8 \\
-10.0 \\
0.0 \\
25.0^{d} \\
-20.0 \\
-15.1 \\
-10.0 \\
25.0^{4} \\
-10.0 \\
0.0 \\
25.0 \\
-10.0 \\
0.0 \\
25.0 \\
25.0^{\circ} \\
-19.9 \\
-10.0 \\
0.0 \\
25.0^{d} \\
-19.9 \\
-15.1 \\
-10.0 \\
25.0^{d}\end{array}$ & $\begin{array}{l}(1.91 \pm 0.03) \times 10^{-2} \\
(2.76 \pm 0.04) \times 10^{-1} \\
(1.85 \pm 0.02) \times 10^{-2} \\
(6.29 \pm 0.04) \times 10^{-2} \\
(1.77 \pm 0.05) \times 10^{-1} \\
2.0 \\
(7.07 \pm 0.02) \times 10^{-2} \\
(1.25 \pm 0.02) \times 10^{-1} \\
(2.17 \pm 0.02) \times 10^{-1} \\
6.1 \\
(1.40 \pm 0.01) \times 10^{-3} \\
(4.73 \pm 0.01) \times 10^{-3} \\
(6.56 \pm 0.01) \times 10^{-2} \\
(1.17 \pm 0.02) \times 10^{-2} \\
(3.78 \pm 0.11) \times 10^{-2} \\
(5.13 \pm 0.05) \times 10^{-1} \\
8.3 \times 10^{-1} \\
(1.54 \pm 0.02) \times 10^{-2} \\
(5.47 \pm 0.02) \times 10^{-2} \\
(1.66 \pm 0.03) \times 10^{-1} \\
2.1 \\
(6.44 \pm 0.03) \times 10^{-2} \\
(1.16 \pm 0.03) \times 10^{-1} \\
(2.05 \pm 0.01) \times 10^{-1} \\
6.7\end{array}$ & $\begin{array}{l}16.7 \\
15.2\end{array}$ & $\begin{array}{l}-8.4 \\
-5.2\end{array}$ \\
\hline
\end{tabular}

"Determined conductimetrically at least in duplicate; errors shown are average deviations. ${ }^{b}$ Solvents are $\% \mathrm{v} / \mathrm{v}$ organic solvent-water. 1 cal $=4.184$ J. "Calculated from rate constants at lower temperatures. "Estimated for the tosylate (ref. 12)-our rate constant for solvolysis of the tosylate is $5.1 \times 10^{-1} \mathrm{~s}^{-1}$, the same as for the mesylate, assuming a OTs/OMs rate ratio of 1.0 [ref. 11(a)].

Table 2 Rate constants $(k)$ for solvolyses of cyclohex-2-en-1-yl mesylate $2^{\circ}$

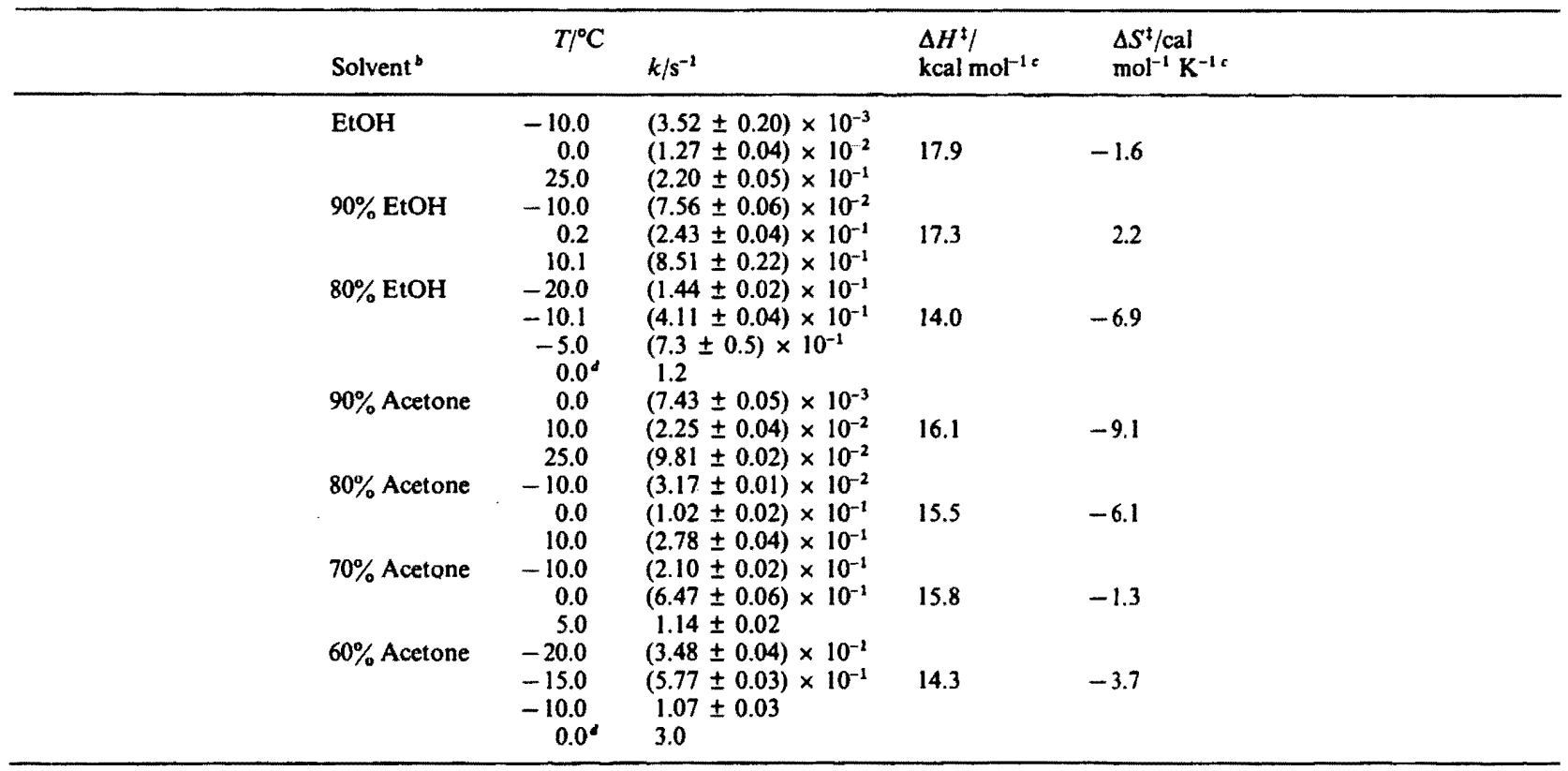

${ }^{a-4}$ As for Table 1 .

Additional rate constants for solvolyses of 3-bromocyclohexene 5 are given in Table 5.

In the rapid-injection kinetic method, a cold, dilute solution of the mesylate is injected into a rapidly-stirred thermostatted solvolysis solvent, and the progress of reaction is monitored by conductimetric methods. In favourable cases, about half of the total increase in conductance occurs during the mixing time ( $\dot{c} a$. $0.5 \mathrm{~s}$ ), because some solvolysis occurs and triethylammonium chloride (a coproduct in the synthesis) and alkyltriethyl- ammonium salts (from reaction of the mesylates with triethylamine) are present. Occurrence of these reactions (summarised in Scheme 1) has been deduced from the spectroscopic and chromatographic evidence given in the Experimental section.

Rate constants (Tables 1-4) were obtained from the changes in conductance after the mixing time. A small batch of mesylate, prepared from alcohol, methanesulfonyl chloride and triethylamine in dichloromethane, may be used in aliquots for several 
Table 3 Rate constants $(k)$ for solvolyses of diphenylmethyl mesylate $3 *$

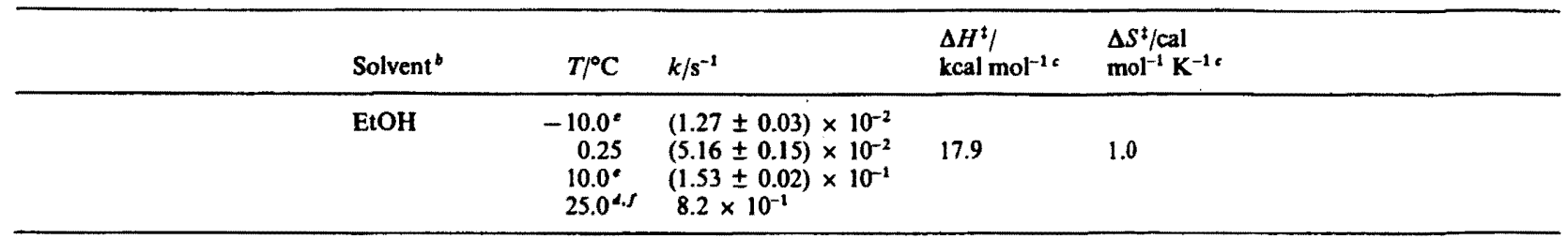

at As for Table 1. "The results at -10 and $+10^{\circ} \mathrm{C}$ were repeated satisfactorily in duplicate in a second batch of solvent. $/$ Six direct measurements at $25^{\circ} \mathrm{C}$ gave $k=(8.6 \pm 1.7) \times 10^{-1} \mathrm{~s}^{-1}$.

Table 4 Rate constants $(k)$ for solvolyses of 1-phenylethyl mesylate $4^{*}$

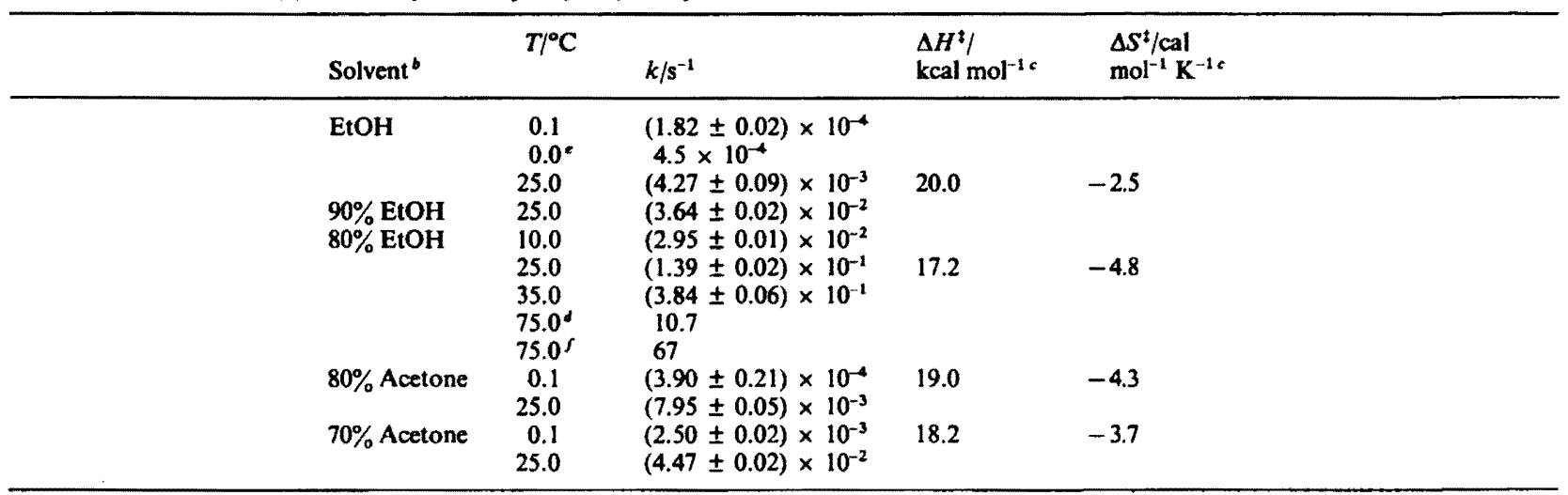

$\because$ As for Table 1. ${ }^{\circ}$ Data for tosylate from ref. 6. 5 Estimated by D. S. Noyce et al. (ref. 10), using a value of $3 \times 10^{4}$ for the $\mathrm{OMs} / \mathrm{Cl}$ rate ratio.

Table 5 Rate constants $(k)$ for solvolyses of 3-bromocyclohexene $5^{\circ}$

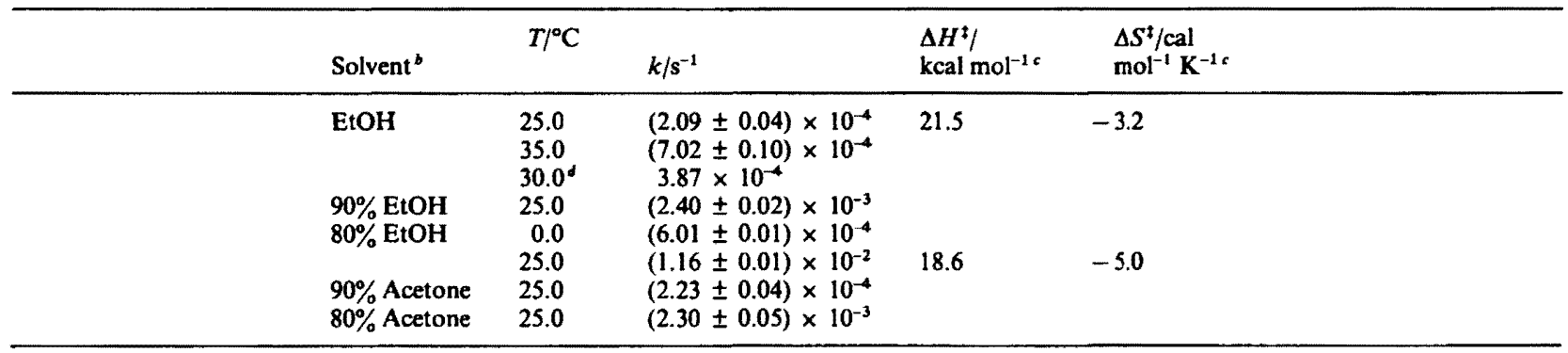

ar As for Table 1. "Interpolated for a comparison given in Table 10.

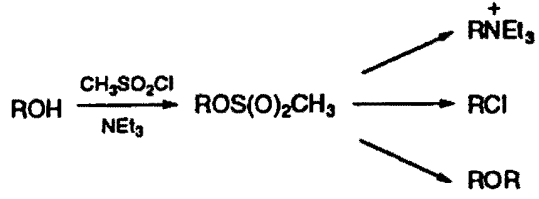

Scheme 1

kinetic runs. An aliquot of dichloromethane solution was evaporated and the mesylate was usually dissolved in cold acetonitrile prior to injection into the solvolysis medium (acetonitrile solutions mix much more rapidly with typical solvolysis media than dichloromethane solutions). If the mesylate decomposed on storage in dichloromethane at $c a$. $-10^{\circ} \mathrm{C}$, the change in conductance during the mixing time increased and there was a relatively small change in conductance during solvolysis. These results can be explained in part by the reaction of excess triethylamine with the mesylates to give alkyltriethylammonium salts (Scheme 1), and also by partial hydrolysis of the mesylate by adventitious water (see Experimental section).

The results reported here (Tables 1-3) refer to the most reactive mesylates ever studied in protic solvolytic media (tosylates react at very similar rates). ${ }^{11}$ Diphenylmethyl mesylate 3, which was studied previously by Bentley et al. ${ }^{3}$ reacts in ethanol at $25^{\circ} \mathrm{C}$ with a rate constant of $8.2 \times 10^{-1} \mathrm{~s}^{-1}$ (Table 3)-eight times faster than the ethanolysis of any mesylate previously investigated by a reliable method. Although direct measurements at $25^{\circ} \mathrm{C}$ were not as reproducible as we had hoped, a short extrapolation from measurements at lower temperatures gives reliable data (Table 3). Diphenylmethyl and 1-phenylethyl tosylates have been examined previously; 5,6 the latter, relatively unreactive compound is reported ${ }^{6}$ to react with ethanol at $0^{\circ} \mathrm{C}$ with $k=4.5 \times 10^{-4} \mathrm{~s}^{-1}$, in fair agreement with the value of $3.3 \times 10^{-4}$ predicted from our mesylate data (Table 4 ), assuming a tosylate/mesylate rate ratio of $1.8 .^{11 a}$ The similarly predicted $k$ value at $25^{\circ} \mathrm{C}$ of $7.7 \times 10^{-3} \mathrm{~s}^{-1}$ is in good agreement with an interpolated $k=8.3 \times 10^{-3} \mathrm{~s}^{-1}$ for ethanolysis of 1-phenylethyl tosylate. ${ }^{6}$

Neither mesylates 1 and 2, nor the corresponding tosylates, have been investigated previously by reliable kinetic methods. Solvolyses of $p$-methoxybenzyl tosylate are of considerable interest because the methoxy substituent lies at the extremity of a curved Hammett plot. ${ }^{12}$ Our estimated rate constant of $5.1 \times 10^{-1} \mathrm{~s}^{-1}$ in $80 \%$ acetone at $25^{\circ} \mathrm{C}$ (see Table 1 , footnote $e$ ) is in fair agreement with a previous less direct estimate of $8.3 \times 10^{-1} \mathrm{~s}^{-1} .12$

Cyclohex-2-en-1-yl mesylate 2 was investigated independ- 
ently by three of us. Measurements in ethanol at $25^{\circ} \mathrm{C}$ were relatively straightforward, but the reproducibility of data for $80 \%$ ethanol-water at $0{ }^{\circ} \mathrm{C}$ was disappointing; the data in Table 2 are the most consistent set.

Although the initial synthesis of the mesylates (Scheme 1) is a clean and rapid process, a study of solvolysis products is complicated by the presence of competing nucleophiles (chloride and triethylamine as well as traces of water). Complications arising from the formation of both elimination and substitution products were avoided by restricting product studies to two substrates for which elimination was impossible. Substitution products of solvolyses of a mesylate (ROMs) in ethanol-water are the ether (ROEt) and the alcohol (ROH). Product ratios and product selectivities [S, defined by eqn. (1)] for solvolyses of $p$-methoxybenzyl mesylate are given in Table 6. The product of ethanolysis of diphenylmethyl mesylate was shown to be the expected ethyl ether.

$$
S=[\text { ROEt }] /[\mathrm{ROH}] \times[\text { water }] /[\text { ethanol }]
$$

\section{Discussion}

Mechanisms of Solvolyses.-Substrates 1-4 are activated towards $S_{N} 1$ reactions by the substituents capable of stabilising positive charge. Even solvolyses of the much less reactive chlorides (see below), are believed to be $S_{N} l$ in character, supported by the observation of common ion rate depression: e.g. p-methoxybenzyl chloride shows common ion rate depression in $50 \% \mathrm{v} / \mathrm{v}$ trifluoroethanol-water, ${ }^{13 a}$ and chlorodiphenylmethane shows common ion rate depression in $80 \%$ acetone-water. ${ }^{13 b}$ The 1-phenylethyl cation is unselective in competition between azide and water, consistent with an extremely short-lived cationic intermediate. ${ }^{13 c}$

Our rate data support the above interpretation. Logarithms of rate constants in any solvent $(k)$ relative to rate constants in $80 \%$ ethanol-water $\left(k_{0}\right)$ can be plotted against $Y$ values defined by $\log \left(k / k_{0}\right)$ for an appropriate model compound [eqn. (2)]. ${ }^{11 a}$

$$
\log \left(k / k_{0}\right)_{\mathbf{R X}}=m Y_{\mathbf{X}}+c
$$

The high slopes of the correlations (Table 7) are consistent with a large amount of positive charge developed in the transition

Table 6 Product selectivities [eqn. (1)] for solvolyses of $p$-methoxybenzyl mesylate 1 in alcohol-water mixtures at $0^{\circ} \mathrm{C}^{a}$

\begin{tabular}{lll}
\hline Solvent & Ethanol-water & Methanol-water \\
\hline 90 & $1.6(1.7)$ & $4.0(3.6)$ \\
80 & $2.0(2.0)$ & $8.8^{\circ}(3.7)$ \\
70 & $2.7(2.3)$ & $(4.0)$ \\
60 & $2.8(2.7)$ & $(4.8)$ \\
\hline
\end{tabular}

- Values in parentheses refer to data from ref. $15 a$ for solvolyses of $p$ methoxybenzyl chloride at $25^{\circ} \mathrm{C}$. ${ }^{\circ}$ Anomalous value probably because of mixing problems. state. Incidentally, the good linear fit (Table 7) and the consistent trends in activation parameters (Tables 1-4) support the reliability of the kinetic data.

The intercepts of eqn. (2), measuring the dispersion of the correlation lines for ethanol-water mixtures from the acetonewater lines, ${ }^{14}$ are explained by differences in solvation at the reaction site between the $\pi$-bonded substrates $1-4$ and the saturated adamantyl model compound defining the $\boldsymbol{Y}_{X}$ scale. ${ }^{14 c, d}$ The results (Table 7) show significant negative intercepts for solvolyses in acetone-water mixtures, as would be expected by analogy with previous work on solvolyses of chlorides. $^{14}$

Selectivity values $[S$, eqn. (1)] for solvolyses of $p$-methoxybenzyl mesylate 1 in $60-90 \%$ ethanol-water are very similar to those for solvolyses of $p$-methoxybenzyl chloride (Table 6), ${ }^{13 a}$ as expected for $S_{N} 1$ reactions via free cations with minimal involvement of the leaving group in the product-determining step. The scope of the results is limited because of mixing problems, particularly in methanol-water mixtures where high $S$ values were observed (Table 6). We have previously found that for very rapid reactions in more aqueous media, erroneously high $S$ values may be obtained. ${ }^{15 b}$ Higher $S$ values were obtained when cold solutions of mesylate in dichloromethane were injected instead of acetonitrile solutions. Presumably dichloromethane disperses more slowly than acetonitrile and most of the reaction may take place in a microphase comprising mainly dichloromethane and ethanol.

Leaving Group Effects.-The new solvolysis rate data (Tables $1-5)$ can be compared with previously published data to expand knowledge of leaving group effects. Mesylate/bromide rate ratios (Table 8) show a relatively low value of 112 for solvolyses of 2-adamantyl derivatives in highly aqueous media at $75^{\circ} \mathrm{C}$. However, in ethanol (a solvent of lower ionizing power) this ratio would be at least three-fold greater because $m$ values for bromides are over 0.1 higher than for mesylates and tosylates (comparing solvolyses of different substrates on one $Y$ scale). ${ }^{11 a .17}$ Also, the effect of a lower temperature would be to increase the $\mathrm{OMs} / \mathrm{Br}$ rate ratio because activation energies for solvolyses of bromides are likely to be greater than for solvolyses of the corresponding, more reactive, tosylates.

The above estimates indicate that a $\mathrm{OMs} / \mathrm{Br}$ rate ratio for solvolyses of 2-adamantyl substrates in ethanol at $25^{\circ} \mathrm{C}$ may be within the range $(600-1600$, Table 8$)$ observed for the other $\mathrm{S}_{\mathrm{N}} \mathrm{l}$ ethanolyses of primary and secondary substrates (Table 8). A previously reported ${ }^{18} \mathrm{OMs} / \mathrm{Br}$ ratio of 231 for solvolyses of 2-adamantyl substrates in $80 \%$ ethanol at $25^{\circ} \mathrm{C}$ may be too low because the bromide data were extrapolated from data at over $100^{\circ} \mathrm{C}$; because precise Arrhenius plots are curved, linear extrapolations give high estimates. ${ }^{3.19}$ There is a small solvent dependence of $\mathrm{OMs} / \mathrm{Br}$ rate ratios, even for solvents of similar ionizing power, as shown by the decrease from 1050 in ethanol to 440 in $90 \%$ acetone-water for solvolyses of 2 (data from Tables 2 and 5).

The main factor influencing $\mathrm{OMs} / \mathrm{Br}$ and $\mathrm{OTs} / \mathrm{Br}$ rate ratios

Table 7 Correlations of rate constants for solvolyses with $Y_{\mathrm{X}}$ values based on the modified Grunwald-Winstein equation [eqn. (2)] ${ }^{*}$

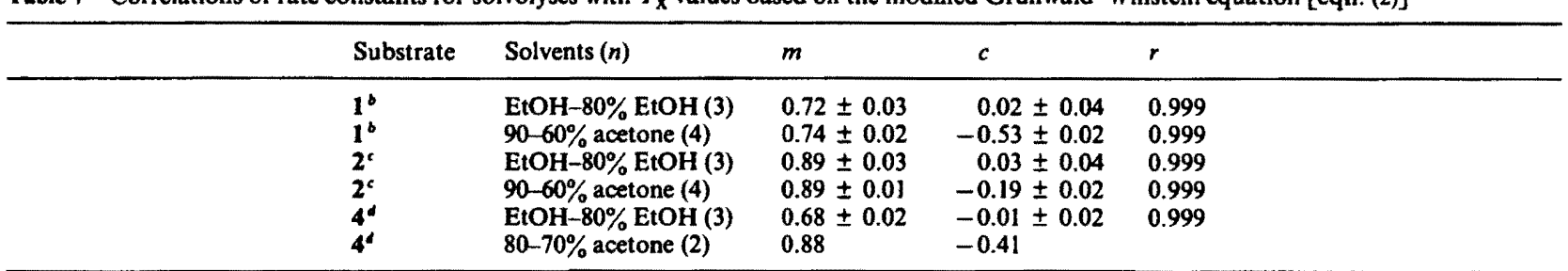

$Y_{\mathrm{X}}$ values from ref. $11 a ;(n)$ is the number of solvents, $m$ and $c$ are defined as in eqn. (2) and $r$ is the correlation coefficient. ${ }^{b}$ Temperature $-10.0^{\circ} \mathrm{C}$; kinetic data from Table 1. " Temperature $0.0^{\circ} \mathrm{C}$; kinetic data from Table 2 . " Temperature $25.0^{\circ} \mathrm{C}$; kinetic data from Table 4. 
Table 8 Mesylate/bromide ( $\mathrm{OMs} / \mathrm{Br})$ rate ratios

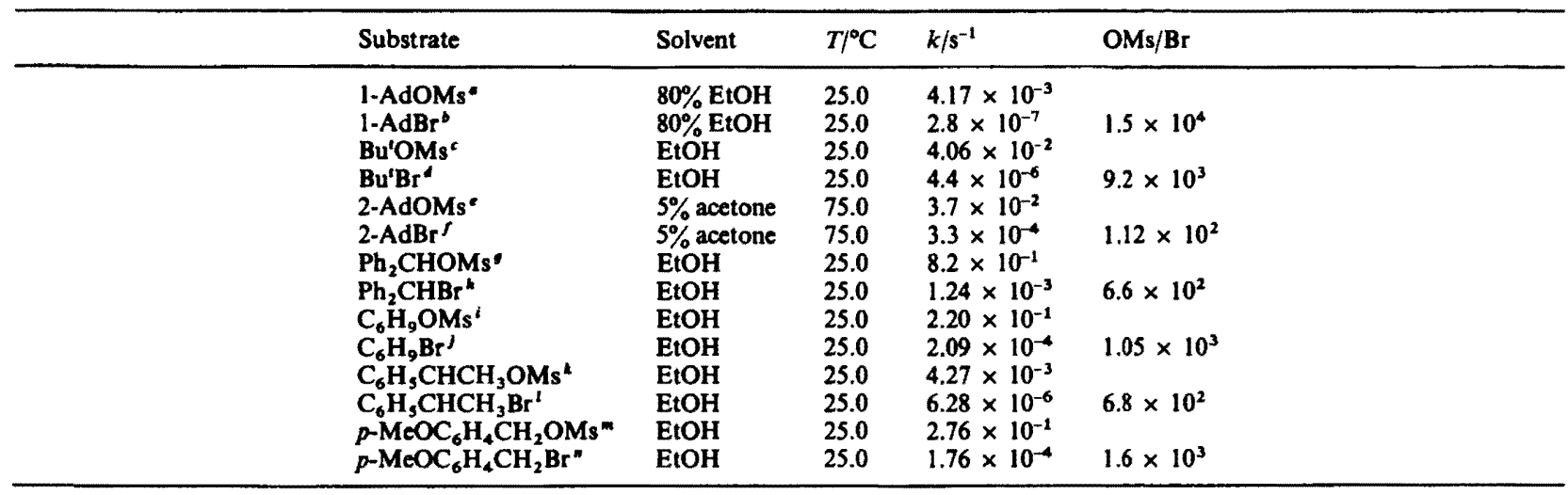

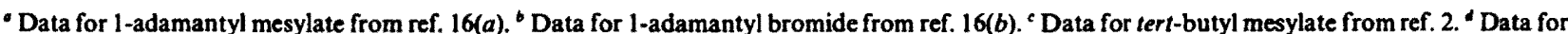
tert-butyl bromide from ref. $16(\mathrm{c})$. 'Data from ref. $16(d)$ for 2-adamantyl mesylate (extrapolated from data at $\left.25-50^{\circ} \mathrm{C}\right) .{ }^{f}$ Data for 2-adamantyl bromide determined by C. T. Bowen (Swansea). ' Data for diphenylmethyl mesylate from Table 3. ${ }^{k}$ Data for bromodiphenylmethane from ref. 14(a).

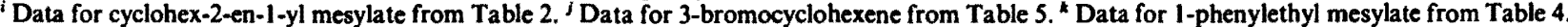
'Data for 1-bromo-1-phenylethane from ref. 16(c). " Data for p-methoxybenzyl mesylate from Table 1. " Data for $p$-methoxybenzyl bromide from ref. 16(e).

Table 9 Temperature dependence of mesylate/p-nitrobenzoate rate ratios

\begin{tabular}{lllll}
\hline Substrate & $25^{\circ} \mathrm{C}$ & $50^{\circ} \mathrm{C}$ & $75^{\circ} \mathrm{C}$ & $100^{\circ} \mathrm{C}$ \\
\hline 1-Phenylethyl & $\left(6.6 \times 10^{9}\right)$ & $1.4 \times 10^{9}$ & $3.7 \times 10^{8}$ & $\left(1.2 \times 10^{8}\right)$ \\
Cyclohex-2-en-1-yl $^{\circ}$ & $\left(4.3 \times 10^{9}\right)$ & $\left(9.5 \times 10^{8}\right)$ & $\left(3.6 \times 10^{8}\right)$ & $\left(8.6 \times 10^{7}\right)$ \\
Diphenylmethyl $^{8}$ & $3.3 \times 10^{9}$ & $9.0 \times 10^{8}$ & $3.0 \times 10^{8}$ & $1.1 \times 10^{8}$ \\
1-Adamantyl $^{8}$ & $2.6 \times 10^{9}$ & $6.2 \times 10^{8}$ & $1.9 \times 10^{8}$ & $6.4 \times 10^{7}$
\end{tabular}

- Values in parentheses are probably less reliable because they include an extrapolation of rate constants over more than a $50^{\circ} \mathrm{C}$ range of temperature. ${ }^{6}$ In $70 \%$ acetone-water; mesylate data from Table 4; $p$-nitrobenzoate data from ref. $23(a)$ extrapolated from data at $99{ }^{\circ} \mathrm{C}$ and $120^{\circ} \mathrm{C}$. ${ }^{\circ}$ In $60 \%$ acetone-water; mesylate data from Table 2; $p$-nitrobenzoate data from ref. $23(b)$ extrapolated from data at 75 and $95{ }^{\circ} \mathrm{C}$. ${ }^{4}$ In $80 \%$ ethanol-water; mesylate and $p$-nitrobenzoate data from ref. 3; $p$-nitrobenzoate data extrapolated from data at 60,75 and $100{ }^{\circ} \mathrm{C} .{ }^{\circ}$ In $60 \%$ ethanol-water; mesylate and $p$-nitrobenzoate data from ref. 3; p-nitrobenzoate data extrapolated from data at 102 and $129^{\circ} \mathrm{C}$.

Table 10 Rate constants for solvolyses of chlorides and bromide/ chloride rate ratios ${ }^{2}$

\begin{tabular}{lllll}
\hline Substrate & Solvent & $T / 7^{\circ} \mathrm{C}$ & $k / \mathrm{s}^{-1}$ & $\mathrm{Br} / \mathrm{Cl}$ \\
\hline 1-AdCl & EtOH & 25.0 & $\mathrm{ca} .2 .5 \times 10^{-11}$ & $\mathrm{ca} .40$ \\
$\mathrm{Bu}^{\prime} \mathrm{Cl}^{\circ}$ & EtOH & 25.0 & $8.6 \times 10^{-8}$ & 51 \\
$\mathrm{Ph}_{2} \mathrm{CHCl}^{\alpha}$ & EtOH & 25.0 & $5.4 \times 10^{-5}$ & 23 \\
$\mathrm{C}_{6} \mathrm{H}_{9} \mathrm{Cl}^{*}$ & EtOH & 30.0 & $1.86 \times 10^{-5}$ & 21 \\
$\mathrm{C}_{6} \mathrm{H}_{5} \mathrm{CHCH}_{3} \mathrm{Cl}^{f}$ & EtOH & 25.0 & $2.16 \times 10^{-7}$ & 29 \\
$p-\mathrm{MeOC}_{6} \mathrm{H}_{4} \mathrm{CH}_{2} \mathrm{Cl}^{\circ}$ & EtOH & 25.0 & $1.67 \times 10^{-5}$ & 10.5 \\
\hline
\end{tabular}

Rate constants for bromides from Table 8 , except where stated otherwise. "Data from ref. $16(b)$. "Data from ref. $11(b)$. $d$ Data from ref. 14(a). "Chloride data from ref. 24(a), and bromide data from Table 5; also $k=8.4 \times 10^{-4} \mathrm{~s}^{-1}$ in $70 \% \mathrm{v} / \mathrm{v}$ acetone-water [ref. $\left.24(b)\right] .{ }^{f}$ Data from ref. $14(b)$. Data from ref. $16(e)$

appears to be electronic effect(s) on the stability of the transition state, as proposed by Hoffmann. ${ }^{6}$ High ratios $(600-1600)$ in ethanol at $25^{\circ} \mathrm{C}$ appear to be indicative of a high degree of charge separation between the carbon and the leaving group in the transition state, with a low degree of bond-making to the carbon by an incoming group. ${ }^{\text {6b }}$ Such charge development in the leaving group does not appear to be transmitted strongly to substituents in the aromatic ring of arenesulfonates because $\rho$ values are in the range 1.3 to 1.9 for both $S_{\mathrm{N}} 1$ and $S_{\mathrm{N}} 2$ solvolyses. ${ }^{20}$

Higher $\mathrm{OMs} / \mathrm{Br}$ rate ratios $\left(\mathrm{ca} .10^{4}\right)$ for the tertiary substrates (1-adamantyl and tert-butyl) are consistent with a steric acceleration of mesylate solvolyses due to the unavoidable interactions between the $\beta$-hydrogens and the oxygen atoms of the sulfonyl group. ${ }^{18}$ Even higher OTs/Br ratios were observed for solvolyses of more hindered substrates: $9 \times 10^{4}$ for bis- trifluoromethyl derivative $6^{21 a}$ and $1.6 \times 10^{6}$ for $7 .^{216}$ The $F$ strain effects in solvolyses of halides and sulfonate esters have recently been investigated; ${ }^{22 a}$ earlier work revealed small but detectable $F$-strain effects in solvolyses of tertiary halides. ${ }^{22 b}$

OMs/OPNB rate ratios (Table 9) are very large (ca. $3 \times 10^{9}$ at $25^{\circ} \mathrm{C}$ ) but, remarkably, vary only three-fold (almost within experimental error) for the above three secondary substrates and the tertiary 1-adamantyl system. It appears that the steric requirements of the carboxylic and sulfonate esters are very similar.

Bromide/chloride rate ratios (Table 10) are slightly higher for solvolyses of tertiary substrates, and solvolyses of $p$-methoxybenzyl halides give the lowest $\mathrm{Br} / \mathrm{Cl}$ rate ratio; the latter is not consistent with geminal electronic effects. Geminal effects have been clearly shown to stabilise the initial state of the reactant when two electronegative substituents are attached directly to the reaction site $\left(\right.$ e.g. $\left.\mathrm{YCH}_{2} \mathrm{X}\right),{ }^{25 b}$ and such effects cause greater stabilisation of the initial states in the order: first row element $>$ second row element etc. ${ }^{26}$ If solvolyses of $p$ methoxybenzyl chloride were subject to geminal effects due to an interaction between the $p$-methoxyphenyl substituent and the halide leaving group, as considered (not proposed) recently, ${ }^{25 b}$ a high $\mathrm{Br} / \mathrm{Cl}$ rate ratio would be expected. Also, the $\mathrm{OMs} / \mathrm{Br}$ rate ratio of 1600 is the highest of those shown in Table $\mathbf{8}$ for primary and secondary substrates, whereas a low value would be expected if geminal effects stabilised the initial state in the order OMs $>$ Br. Hence, our data indicate that the phenyl ring effectively insulates the p-methoxy-substitutent from the geminal electronic interactions ${ }^{25,26}$ with $\alpha$-substituents (e.g. leaving groups) of $p$-methoxyphenyl derivatives. $F$-Strain effects for iodides may be larger than for mesylates in 
Table 11 A scale of leaving group $(X)$ effects for $S_{N} 1$ reactions based on solvolyses of 1-phenylethyl substrates in $80 \%$ ethanol-water at $75^{\circ} \mathrm{C}$ updated from Noyce et al. (ref. 10)

\begin{tabular}{lll}
\hline $\mathrm{X}^{-}$ & $k / \mathrm{s}^{-1}$ & Rel. rate \\
\hline $\mathrm{CH}_{3} \mathrm{C}(\mathrm{O}) \mathrm{O}^{-c}$ & $1 \times 10^{-10}$ & $5 \times 10^{-8}$ \\
$\mathrm{C}_{6} \mathrm{H}_{5} \mathrm{C}(\mathrm{O}) \mathrm{O}^{-6}$ & $5.5 \times 10^{-10}$ & $2.8 \times 10^{-7}$ \\
$4-\mathrm{NO}_{2} \mathrm{C}_{6} \mathrm{H}_{4} \mathrm{C}(\mathrm{O}) \mathrm{O}^{-c}$ & $1.2 \times 10^{-8}$ & $6.2 \times 10^{-6}$ \\
$3,5-\left(\mathrm{NO}_{2}\right)_{2} \mathrm{C}_{6} \mathrm{H}_{3} \mathrm{C}(\mathrm{O}) \mathrm{O}^{-4}$ & $7.2 \times 10^{-8}$ & $3.7 \times 10^{-5}$ \\
$\mathrm{Cl}^{-}$ & $1.94 \times 10^{-3}$ & 1 \\
$\mathrm{CF}_{3} \mathrm{C}(\mathrm{O}) \mathrm{O}^{-5}$ & $5.54 \times 10^{-3}$ & 2.9 \\
$\mathrm{O}_{2} \mathrm{NO}^{-}$ & $6.9 \times 10^{-3}$ & 3.6 \\
$\mathrm{Br}^{-k}$ & $2.98 \times 10^{-2}$ & 15.4 \\
$1^{-1}$ & $6.0 \times 10^{-2}$ & 30.9 \\
$\mathrm{CH}_{3} \mathrm{~S}(\mathrm{O})_{2} \mathrm{O}^{-j}$ & 10.7 & $5.5 \times 10^{3}$ \\
$4-\mathrm{CH}_{3} \mathrm{C}_{6} \mathrm{H}_{4} \mathrm{~S}(\mathrm{O})_{2} \mathrm{O}^{-k}$ & 10.7 & $5.5 \times 10^{3}$ \\
$4-\mathrm{NO}_{2} \mathrm{C}_{6} \mathrm{H}_{4} \mathrm{~S}(\mathrm{O})_{2} \mathrm{O}^{-1}$ & $4.4 \times 10^{2}$ & $2.3 \times 10^{5}$ \\
$\mathrm{CF}_{3} \mathrm{~S}(\mathrm{O})_{2} \mathrm{O}^{-m}$ & $2.2 \times 10^{6}$ & $1.1 \times 10^{9}$ \\
\hline
\end{tabular}

- Calculated from data in $30 \%$ ethanol-water extrapolated to $75^{\circ} \mathrm{C}$ [ref. $27(a)$ ], and then further corrected to $80 \%$ ethanol-water by a factor of $c a$. $10^{3}$. ${ }^{\circ}$ Calculated by dividing the rate constant for $4-\mathrm{NO}_{2} \mathrm{C}_{6} \mathrm{H}_{4}$ $\mathrm{C}(\mathrm{O}) \mathrm{O}^{-}$by a factor of 22 (ref. 3 ). 'Calculated from extrapolated data in $70 \%$ acetone-water [ref. 23(a)]; further correction to $80 \%$ ethanolwater was not required because $Y_{\text {or, }}$, for $70 \%$ acetone is 0.07 [ref. $11(a)$ ]. ${ }^{4}$ Calculated by multiplying the rate constant for $4-\mathrm{NO}_{2} \mathrm{C}_{6} \mathrm{H}_{4} \mathrm{C}(\mathrm{O}) \mathrm{O}^{-}$ by a factor of 6 (ref. 3). Extrapolated data from ref. 14(b). ${ }^{-}$Ref. 10. - Calculated from data in 90 and $60 \%$ ethanol-water [ref. $27(b)$ ] by interpolation of a plot of logarithms of rate constants vs. $\mathrm{Y}\left(\mathrm{OClO}_{3}\right)$ [ref. $11(a)]$. "Extrapolated data from ref. $16(c)$. ${ }^{i}$ Calculated assuming a I/Br rate ratio of 2, as observed for 1-adamantyl solvolyses [ref. 27(c)]. 'Data from Table 4. "Calculated from rate data for the mesylate assuming a OTs/OMs rate ratio of 1.0 for solvolyses in $80 \%$ ethanolwater [ref. 11(a)]. ' Calculated from rate data for the tosylate using the Hammett equation and assuming a reaction constant $\rho=1.7$ for solvolyses in $80 \%$ ethanol-water at $75^{\circ} \mathrm{C}$ (ref. 20). ${ }^{m}$ Calculated from rate data for the tosylate assuming a triflate/tosylate rate ratio of $2 \times 10^{5}$, as observed for solvolyses of 2 -adamantyl sulfonates in $80 \%$ ethanol-water at $75^{\circ} \mathrm{C}$; kinetic data for solvolyses of 2-adamantyl tosylate from ref. $27(d)$ and extrapolated kinetic data for 2-adamantyl triflate from ref. 17.

special cases, ${ }^{22 a}$ so one possible explanation of the low $\mathrm{Br} / \mathrm{Cl}$ rate ratio for solvolyses of the primary substrate $p$-methoxybenzyl chloride is $F$-strain increasing the $\mathrm{Br} / \mathrm{Cl}$ rate ratios for solvolyses of the secondary and tertiary bromides.

The relatively consistent results for leaving group effects in unhindered substrates are in marked contrast to the recent dire warnings about deducing carbocation stabilities from solvolysis rate constants. ${ }^{25 a, 25 b}$ It appears that rates of acid catalysed $\mathrm{OH}$ exchange in alcohols and of solvolyses of fluorides may be strongly susceptible to geminal effects stabilising the initial states, ${ }^{25 b}$ leading to anomalously low solvolytic reactivities. However, these systems are atypical sources of solvolytic data, and solvolyses of mesylates and p-nitrobenzoates (both having an oxygen atom as the first row element attached to the reaction site) give remarkably consistent leaving group effects (Table 9).

Solvolyses of 1 -phenylethyl substrates in $80 \%$ ethanol-water at $75^{\circ} \mathrm{C}$ provide the most extensive data set available for leaving group effects on $S_{N} 1$ reactions, ${ }^{10}$ although such a wide range (over $10^{16}$ in rate) cannot be determined directly. Our value for solvolysis of 1 -phenylethyl mesylate in $80 \%$ ethanol-water at $75^{\circ} \mathrm{C}$ (Table 4) differs five-fold from the previous estimate, ${ }^{10}$ and provides a revised anchor point from which new values for sulfonates can be calculated (Table 11); additional and revised values for carboxylates are also given in Table 11 .

Reliability and Scope of the Synthesis of Unstable Mesylates. - Comparing rate data for chlorides (Table 10) with data for corresponding mesylates (Tables $1-4$ ), the relative rates differ by over $10^{4}$. Consequently, although chlorides may be formed in low yields during the synthesis and/or manipulation of the unstable mesylates (e.g. for diphenylmethyl and cyclohex2-en-1-yl mesylates-see Experimental section), the accuracy of the first order solvolysis rate constants for the mesylates (Tables.1-4) would not be significantly affected by a concurrent solvolysis even of large amounts of chloride impurities (only small amounts are likely to be present).

The scope for the synthesis of unstable mesylates can be predicted from the solvolysis rate constants for the corresponding chlorides, data for many of which are available in the literature. Kinetic data for over 300 solvolyses of chlorides and bromides under diverse reaction conditions have recently been interpolated or extrapolated to standard conditions-solvolysis of chlorides in pure ethanol at $25^{\circ} \mathrm{C} . .^{28}$ Solvolysis rate constants under these conditions (Table 10) show the reactivities of chloride substrates for which unstable mesylates can be synthesised and characterised in cooled solutions; i.e. primary and secondary alcohols, whose chloride derivatives have $k \leqslant 5 \times 10^{-5} \mathrm{~s}^{-1}$ (Table 10), probably can be derivatised as unstable mesylates. Assuming that $\mathrm{OMs} / \mathrm{Cl}$ rate ratios for tertiary substrates are ten-fold higher than for secondary substrates (see above), it should be possible to convert a tertiary alcohol to an unstable mesylate if ethanolysis of the corresponding chloride at $25^{\circ} \mathrm{C}$ had $k<5 \times 10^{-6} \mathrm{~s}^{-1}$.

1-Phenylethyl mesylate 4 , the least reactive mesylate described in this work, is reported ${ }^{8}$ to be a highly reactive liquid which decomposes violently at room temperature. Hence, isolation of these mesylates should be attempted with appropriate caution. Nevertheless, unstable mesylates may be useful reactive intermediates in syntheses, because cold solutions can be prepared economically (avoiding the expense of silver salts). ${ }^{1}$ For nucleophilic displacements, it would be necessary to minimise competition from chloride ion or from triethylamine by adding an excess of the desired nucleophile and/or by precipitating the triethylamine hydrochloride salt.

\section{Conclusions}

Mesylates can be prepared cleanly and rapidly by treatment of methanesulfonyl chloride and triethylamine with alcohols in chlorinated solvents at low temperatures $\left(0\right.$ to $\left.-20^{\circ} \mathrm{C}\right)$, and can be characterised by NMR. Decomposition of mesylates under these conditions leads to chlorides, ethers and tetraalkylammonium salts, but reactions with an excess of added nucleophiles may be of synthetic value.

Microlitre volumes of cold solutions of mesylates in acetonitrile can be injected rapidly into solvolytic media to obtain rate and product data. The results have provided: (i) a revised scale of leaving group effects for $S_{N} 1$ reactions, based on solvolyses of I-phenylethyl substrates in $80 \%$ ethanol-water at $75^{\circ} \mathrm{C}$ (Table 11 ); (ii) further evidence that mesylate/ $p$-nitrobenzoate rate ratios are remarkably independent of the substrate; (iii) further evidence for both ground state steric and transition state electronic effects on $\mathrm{OMs} / \mathrm{Br}$ rate ratios; (iv) evidence from $\mathrm{Br} / \mathrm{Cl}$ rate ratios that geminal electronic effects (stabilising ground states), as considered very recently, ${ }^{25}$ do not appear to influence relative rates of solvolyses of $p$-methoxybenzyl chloride, and also there are no detectable geminal electronic effects for solvolyses of the corresponding bromide, mesylate or $p$-nitrobenzoate.

\section{Experimental}

Materials. - Cyclohex-2-en-1-ol was prepared from 3-bromocyclohexene (Lancaster) by hydrolysis with aqueous sodium carbonate at $25^{\circ} \mathrm{C}$. Diphenylmethanol, 1-phenylethanol and $p$ methoxybenzyl alcohol were commercial samples. Methanesulfonyl chloride was distilled under reduced pressure, and triethylamine and dichloromethane were also distilled before 
use. Acetonitrile was an HPLC grade stored over molecular sieves. Solvents for kinetics and for chromatography were as described previously. ${ }^{29}$

NMR Characterisation of Unstable Mesylates.-A stirred solution of the appropriate alcohol $(1 \mathrm{mmol})$ and triethylamine (152 $\mathrm{mg}, 1.50 \mathrm{mmol})$ in deuteriochloroform $\left(3.5 \mathrm{~cm}^{3}\right)$ was cooled to $-20^{\circ} \mathrm{C}$ under nitrogen. Methanesulfonyl chloride $(126 \mathrm{mg}, 1.1 \mathrm{mmol})$, dissolved in deuteriochloroform $\left(1.5 \mathrm{~cm}^{3}\right)$, was then added dropwise over $10 \mathrm{~min}$. Stirring was continued at $-20^{\circ} \mathrm{C}$, and ${ }^{1} \mathrm{H}$ and ${ }^{13} \mathrm{C}$ NMR spectra (TMS as internal standard) were then taken on a Bruker AC 200 spectrometer; $J$ values are given in $\mathrm{Hz}$.

p-Methoxybenzyl Mesylate 1.-After $30 \mathrm{~min}$ at $-20^{\circ} \mathrm{C}$, an NMR spectrum showed $90 \%$ of the mesylate, with $<5 \%$ unchanged alcohol $\left(\delta=4.56\right.$ for $\left.\mathrm{CH}_{2}\right)$ and with $\mathrm{ca} .5 \%$ of a second impurity $\left(\delta=4.61\right.$ for $\left.\mathrm{CH}_{2}\right)$ assigned to the $p$-methoxybenzyltriethylammonium ion on the basis of NMR (see below). After $12 \mathrm{~h}$ at $+20^{\circ} \mathrm{C}$ NMR showed complete decomposition of the mesylate to the mesylate anion along with the $p$ methoxybenzyltriethylammonium ion ( $c a .70 \%$ and an unidentified coproduct (not the starting alcohol or the chloride, possibly bis-p-methoxybenzyl ether). For $1: \delta_{\mathrm{H}}\left(-20^{\circ} \mathrm{C}\right) 2.91$ $\left(\mathrm{s}, \mathrm{SCH}_{3}\right), 3.85\left(\mathrm{~s}, \mathrm{OCH}_{3}\right), 5.23\left(\mathrm{~s}, \mathrm{CH}_{2}\right), 6.95$ and 7.41 (AA' BB' spectrum, $\mathrm{ArH}) ; \delta_{\mathrm{C}}\left(-20^{\circ} \mathrm{C}\right) 38.4\left(\mathrm{SCH}_{3}\right), 55.3\left(\mathrm{OCH}_{3}\right), 72.4$ $\left(\mathrm{CH}_{2}\right), 114.0(\mathrm{C}-3), 131.1(\mathrm{C}-2), 133.6(\mathrm{C}-1), 160.2(\mathrm{C}-4)$. For $p-$ methoxybenzyltriethylammonium salt: $\delta_{\mathrm{H}}\left(25^{\circ} \mathrm{C}\right) 1.45(\mathrm{t}, J$ 7.3, $\mathrm{CH}_{2} \mathrm{CH}_{3}$ ), 3.34 (q, $\left.\mathrm{CH}_{2} \mathrm{CH}_{3}\right), 3.83\left(\mathrm{~s}, \mathrm{OCH}_{3}\right), 4.57$ (s, $\left.\mathrm{ArCH}_{2}\right), 6.93$ and 7.54 (AA' $\mathrm{BB}^{\prime}$ spectrum, $\mathrm{ArH}$ ).

Cyclohex-2-en-1-yl Mesylate 2.-After $60 \mathrm{~min}$ at $-20^{\circ} \mathrm{C}$, an NMR spectrum showed the complete consumption of cyclohex2-en-1-ol and the formation of 2 and probably 3-chlorocyclohexene in the ratio of $6: 1$. After the sample had been kept at room temperature for $30 \mathrm{~min}$, NMR showed that 2 had been converted completely to 3-chlorocyclohexene. For $2: \delta_{\mathrm{H}} 1.55$ $2.35(\mathrm{~m}, 6 \mathrm{H}), 3.11\left(\mathrm{~s}, \mathrm{CH}_{3}\right), 5.17(\mathrm{br} \mathrm{q}$, line distance $4.2,1-\mathrm{H})$, 5.82 [ddt, $J_{2,3} 10.0, J_{1,2} 4.1, J_{2,4}(2 \times) 2.1,2-H$ ], $6.15\left(\mathrm{dt}, \Sigma J_{3,4}\right.$ 7.2, 3-H); $\delta_{\mathrm{C}} 17.6(\mathrm{t}, \mathrm{C}-5), 24.5(\mathrm{t}, \mathrm{C}-4), 28.8(\mathrm{t}, \mathrm{C}-6), 38.7$ (q, $\mathrm{CH}_{3}$ ), 76.4 (d, C-1), 123.5 (d, C-2), 135.7 (d, C-3).

The product assigned to 3-chlorocyclohexene had $\delta_{\mathrm{H}} 1.55$ $2.20(\mathrm{~m}, 6 \mathrm{H}), 4.65(\mathrm{~m}, 3-\mathrm{H}), 5.73-5.97(\mathrm{~m}, 1-\mathrm{H}$ and $2-\mathrm{H}) ; \delta_{\mathrm{c}}$ 17.9 (t, C-5), 24.1 (t, C-6), 31.9 (t, C-4), 55.1 (d, C-3), 127.5 (d, $\mathrm{C}-2), 130.8(\mathrm{~d}, \mathrm{C}-1)$; these values are shifted 0.1 to $0.9 \mathrm{ppm}$ to higher field than those for an authentic sample of 3-chlorocyclohexene in deuteriochloroform (i.e. no salts present).

Solutions also contained triethylamine in equilibrium with triethylammonium chloride and mesylate; $\delta_{\mathrm{H}}\left(-20 /+25^{\circ} \mathrm{C}\right)$ $1.3 / 1.3$ (t), 2.8/2.7 (s), 2.9/3.1 (q), 10.4/10.2 (br s); $\delta_{\mathrm{C}}(-20 /$ $\left.+27^{\circ} \mathrm{C}\right) 9.5 / 8.7(\mathrm{q}), 39.4 / 39.0(\mathrm{q}), 45.7 / 45.7(\mathrm{t})$.

Alternatively NMR spectra could be obtained in deuteriochloroform above $0^{\circ} \mathrm{C}$ by first synthesising the mesylates at -20 or $-10^{\circ} \mathrm{C}$ in dichloromethane. Most of the triethylammonium salts were separated using a cotton wool plug, and the dichloromethane was evaporated at low temperatures before cold deuteriochloroform was added. The NMR tube was kept at $0^{\circ} \mathrm{C}$ for a few min before the ${ }^{1} \mathrm{H}$ NMR spectrum was obtained using a Perkin-Elmer R24B spectrometer with the probe at ambient temperature. It appears that $p$-methoxybenzyl mesylate 1 is more vulnerable to attack by triethylamine (probably $S_{N} 2$ ) than the other mesylates studied.

Kinetic Method for Unstable Mesylates.-Mesylates were synthesised on a ca. $10 \mathrm{mg}$ scale at -10 or $-20^{\circ} \mathrm{C}$ in dichloromethane, as described above except that a nitrogen atmosphere was not used. All operations involving solutions containing the mesylate were carried out using cooled, insulated syringes or dropping pipettes. After most of the precipitated salt had been removed and the dichloromethane had been evaporated, the mesylate was dissolved in cold acetonitrile at $-20^{\circ} \mathrm{C}$. Under these conditions, solutions of $p$-methoxybenzyl mesylate 1 suitable for the kinetic work could be stored for over $1 \mathrm{~h}$. A small volume of the mesylate solution $\left(10-40 \mathrm{~mm}^{3}\right)$ was then injected into the turbo-stirred conductivity cell as described recently elsewhere. ${ }^{29}$

Chromatographic Analysis of Products.-Cold solutions of mesylates were synthesised as described above, and chromatographic conditions for each substrate were as described recently. ${ }^{15 a}$ When, after $1 \mathrm{~min}$ at $-10^{\circ} \mathrm{C}$, an aliquot of the dichloromethane solution used to prepare diphenylmethyl mesylate 3 was injected into ethanol, HPLC showed complete consumption of alcohol and formation of the corresponding ethyl ether. ${ }^{15 a}$ When another aliquot was injected after $40 \mathrm{~min}$ at $-10^{\circ} \mathrm{C}$, the HPLC analysis showed mainly the ethyl ether but traces of diphenylmethanol and of chloro(diphenyl)methane were also detected.

Solutions of $p$-methoxybenzyl mesylate 1 in dichloromethane at $-20^{\circ} \mathrm{C}$ were obtained and were analysed similarly by quenching in ethanol. After a $5 \mathrm{~min}$ reaction time followed by the quenching of an aliquot, HPLC showed $c a .10 \%$ unchanged alcohol which was reduced to $<3 \%$ after a 20 min reaction time. However, after the transfer to acetonitrile, the percentage of $p$ methoxybenzyl alcohol increased with time; presumably traces of water were solvolysing the mesylate. An alternative possibility would be to store the mesylate in cold pentane, before evaporating the pentane and adding cold acetonitrile. HPLC analysis of the precipitated salt showed a peak at short retention time consistent with the presence of $p$-methoxybenzyltriethylammonium chloride.

For each measurement of product ratios in ethanol-water mixtures, accurate $\mathrm{mm}^{3}$ volumes of solutions of the mesylate in cold acetonitrile were injected (in duplicate) into both pure ethanol and into one of the aqueous ethanol mixtures using the turbostirrer described recently. ${ }^{15 a, 29}$ The $\mathrm{pH}$ of the solution was $c a .3$ and HPLC analyses were carried out with minimum delay (product ratios changed if quenched solutions were left at $0^{\circ} \mathrm{C}$ for several hours); the integrated area corresponding to $p$-methoxybenzyl alcohol in the ethanol quench was subtracted (if necessary) from the area observed for the ethanol-water quench to give the correct amount of p-methoxybenzyl alcohol formed by solvolysis of the mesylate in ethanol-water.

\section{Acknowledgements}

This work was initiated as part of the British-German ARC programme through a grant from the British Council and the Deutscher Akademischer Austauschdienst. We are grateful for additional financial support from the SERC (UK) for the purchase of HPLC equipment, the Deutsche Forschungsgemeinschaft, and the Fonds der Chemischen Industrie (Germany). We are also grateful to D. N. Kevill for helpful discussions during the tenure of a NATO collaborative award (grant CRG. 921261, with T. W. B.), and to H. M. R. Hoffmann and J.P. Richard for helpful comments.

\section{References}

1 K. Takeuchi, K. Ikai, T. Shibata and A. Tsugeno, J. Org. Chem., $1988,53,2852$

2 T. W. Bentley, W. Kirmse, G. Llewellyn and F. Söllenböhmer, J. Org. Chem., 1990, 55, 1536.

3 T. W. Bentley, M. Christl and S. J. Norman, J. Org. Chem., 1991, 56, 6238.

4 T. W. Bentley, S. J. Norman, E. Gerstner, R. Kemmer and M. Christl, Chem. Ber., 1993, 126, 1749. 
5 G. W. H. Cheeseman and R. C. Poller, J. Chem. Soc., 1962, 5277; A. Ledwith and D. G. Morris, J. Chem. Soc, 1964, 508.

6 (a) H. M. R. Hoffmann, J. Chem. Soc., 1965, 6753; (b) 6762.

7 J. K. Kochi and G. S. Hammond, J. Am. Chem. Soc., 1953, 75, 3443.

8 R. K. Crossland and K. L. Servis, J. Org. Chem., 1970, 35, 3195.

9 J. F. King and J. R. du Manoir, J. Am. Chem. Soc., 1975, 97, 2566.

10 D. S. Noyce and J. A. Virgilio, J. Org. Chem., 1972, 37, 2643.

11 (a) T. W. Bentley and G. Llewellyn, Prog. Phys. Org. Chem., 1990, 17, 121 ; (b) A. H. Fainberg and S. Winstein, J. Am. Chem. Soc., 1956, 78, 2770 .

12 M. Fujio, M. Goto, T. Susuki, I. Akasaka, M. Mishima and Y. Tsuno, Bull. Chem. Soc. Jpn., 1990, 63, 1146.

13 (a) T. L. Amyes and J. P. Richard, J. Am. Chem. Soc., 1990, 112,9507; (b) O. T. Benfey, E. D. Hughes and C. K. Ingold, J. Chem. Soc., 1952, 2488; (c) J. P. Richard and W.P. Jencks, J. Am. Chem. Soc., 1982, 104, 4689; J. P. Richard and W. P. Jencks, J. Am. Chem. Soc., 1984, 106, 1373.

14 (a)S. Winstein, A. H. Fainberg and E. Grunwald, J. Am. Chem. Soc., 1957, 79, 4146; (b) A. H. Fainberg and S. Winstein, J. Am. Chem. Soc., 1957, 79, 1597; (c) K.-T. Liu and H. -C. Sheu, J. Org. Chem., 1991, 56, 3021; (d) T. W. Bentley, J.-P. Dau-Schmidt, G. Llewellyn and H. Mayr, J. Org. Chem., 1992, 57, 2387.

15 (a) T. W. Bentley and Z. H. Ryu, J. Chem. Soc., Perkin Trans. 2, 1994, 761 ; (b) T. W. Bentley, H. C. Harris and I. S. Koo, J. Chem. Soc., Perkin Trans. 2, 1988, 783.

16 (a) T. W. Bentley and G. E. Carter, J. Org. Chem., 1983, 48, 579; (b) T. W. Bentley and G. E. Carter, J. Am. Chem. Soc., 1982, 104, 5741; (c) A. H. Fainberg and S. Winstein, J. Am. Chem. Soc., 1957, 79, 1602; (d) T. W. Bentley and C. T. Bowen, J. Chem. Soc., Perkin Trans. 2, 1978, 557; (e) D. A. da Roza, L. J. Andrews and R. M. Keefer, J. Am. Chem. Soc., 1973, 95, 7003.

17 T. W. Bentley and K. Roberts, J. Org. Chem., 1985, 50, 4821.

18 J. L. Fry, C. J. Lancelot, L. K. M. Lam, J. M. Harris, R. C. Bingham, D. J.Raber, R. E. Hall and P. v. R. Schleyer, J. Am. Chem. Soc., 1970, 92, 2538.
19 R. E. Robertson, Prog. Phys. Org. Chem., 1967, 4, 213.

20 D. N. Kevill, K. C. Kolwyck, D. M. Shold and C.-B. Kim, J. Am. Chem. Soc., 1973, 95, 6022.

21 (a) J. P. Richard, T. L. Amyes, L. Bei and V. Stubblefield, J. Am. Chem. Soc., 1990, 112, 9513; (b) K.-T. Liu and M.-Y. Kuo, Tetrahedron Lett., 1985, 26, 355.

22 (a) Y. Ohga and K. Takeuchi, J. Phys. Org. Chem., 1993, 6, 293; (b) H. C. Brown and A. Stern, J. Am. Chem. Soc., 1950, 72, 5068.

23 (a) H. L. Goering, R. G. Briody and G. Sandrock, J. Am. Chem. Soc., $1970,92,7401$; (b) S. S. Kantner, K. Humski and H. L. Goering. J. Am. Chem. Soc., 1982, 104, 1693.

24 (a) H. L. Goering, T. D. Nevitt and E. F. Silversmith, J. Am. Chem. Soc., 1955, 77, 5026; (b) A. S. Bloss, P. R. Brook and R. M. Ellam, J. Chem. Soc., Perkin Trans. 2, 1973, 2165.

25 (a) Y. Apeloig, R. Biton and A. Abu-Freih, J. Am. Chem. Soc., 1993, 115,$2522 ;(b)$ J. P. Richard, T. L. Amyes and D. J. Rice, J. Am. Chem. Soc., 1993, 115, 2523.

26 P. v. R. Schleyer, E. D. Jemmis and G. W. Spitznagel, J. Am. Chem. Soc., 1985, 107, 6393 .

27 (a) E. A. Hill, M. L. Gross, M. Stasiewicz and M. Manion, J. Am. Chem. Soc., 1969, 91, 7381; (b) J. W. Baker and T. G. Heggs, J. Chem. Soc., 1955, 616; (c) T. W. Bentley, G. E. Carter and K. Roberts, J. Org. Chem., 1984, 49, 5183; (d) P. v. R. Schleyer, J. L. Fry, L. K. M. Lam and C. J. Lancelot, J. Am. Chem. Soc, 1970, 92, 2542. 28 (a) J.-P. Dau-Schmidt, Dissertation, Universitat Lübeck, 1992; (b) J.-P. Dau-Schmidt, H. Mayr and T. W. Bentley, in preparation. 29 T. W. Bentley and R. E. Jones, J. Chem. Soc., Perkin Trans. 2, 1993, 2351.

Paper 4/03551H

Received 13th June 1994 Accepted 22nd August 1994 